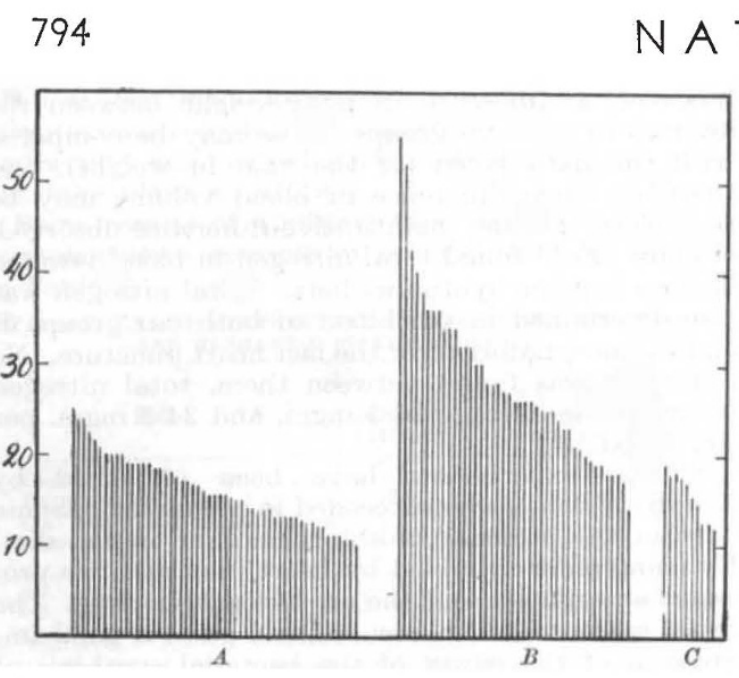

TIME IN MINUTES REQUIRED FOR DECOLORIZATION OF METHYLENE BLDE in vacuo

$A$, controls ; $B$, choline-deficient rats ; $C$, choline-deficient rats which had been given $1 \mathrm{mgm}$. choline chloride intramuscularly 60 minutes before being killed. Every line represents the mean of two values from the same rat. Each Thunberg tube contained

$1.250 \%$ methylene blue, dissolved in $2.5 \mathrm{ml}$. M/15 phosphate buffer $p \mathrm{H} 7 \cdot 15$, and $0 \cdot 2 \mathrm{gm}$. of minced muscle of hind limbs.

determined every day. Every second day, four controls and four 'choline-free' rats were killed and examined for liver fat, blood sugar, bilirubin, nonprotein nitrogen, and prothrombin index. The oxygen consumption of the mineed muscles of the hind limbs was measured in Warburg respirometers.

In accordance with Griffith and Wade ${ }^{2}$ and others, we found renal hæmorrhages on the seventh to ninth day followed by uræmia. At this point there was, as a rule-but not always - a slight increase in liver fat but no increased bilirubin values. Earlier, deposition of fat in the liver was observed, and two to four days before the occurrence of renal hæmorrhages the impeded growth, quite obvious on the fourth day, suggested a disturbance of a more general kind.

Considering the mechanism of action of the better known members of the B vitamins, for example, thiamine, lactoflavine and nicotinic acid, we studied the oxidative metabolism of minced muscles of the hind limbs. In choline-deficient animals oxygen consumption decreased to about two thirds of the normal values. In twenty-five animals on the test diet plus choline the oxygen consumption of $1 \mathrm{gm}$. of muscle pulp in 60 minutes was found to be $56.4 \pm 1.5 \mathrm{~mm} .^{3}$; in twenty-one rats on choline-deficient diet the corresponding value was $37 \cdot 1 \pm 1 \cdot 3 \mathrm{~mm} .^{3}$. The impairment of the metabolism occurred earlier than renal hæmorrhages.

The decrease in oxygen uptake corresponds to a slower decolorization of methylene blue in vacuo (see graph). Addition of choline in physiological concentrations to the minced muscles in vitro is not sufficient to normalize the metabolism. Experiments with addition of $d$-l-methionine in vitro have hitherto not given positive results, in spite of the prominent role of this amino-acid in transmethylation. The specificity of the impairment of the oxidative metabolism as a symptom of choline deficiency is, however, shown by the pharmacological effect of choline. If $1 \mathrm{mgm}$. of choline chloride is injected intra-muscularly only 60 minutes before the choline-deficient animal is used for experiment, the ability of its muscles to decolorize methylene blue is normal.

These experiments suggest that choline, or at least its methyl groups, are essential for intermediary
November 30,1946

Vol. 158

metabolism, probably by being used in the formation of an unknown co-enzyme.

Detailed reports are to be published in Acta Pharmacologica et Toxicologica.

N.-O. ABDON

N. E. Borglin

Department of Physiological Chemistry, University of Lund.

Oct. 29.

'Best, C. H., and Lucas, C. C., "Vitamins and Hormones", 1, 1 (1943).

${ }^{2}$ Griffith, W. H., and Wade, N. J., J. Biol. Chem., 131, 567 (1939); 132, $627(1940)$.

\section{Aggregation of Red Blood Cells in a Strong Electric Field}

ERythrocytes, when suspended in blood plasma, form 'rouleaux', in which the single erythrocytes lie parallel like a pile of coins. This phenomenon is dependent on the presence of a specific substance in the plasma. When suspended in an isotonic sugar solution, the erythrocytes settle to the bottom very slowly, without forming any characteristically shaped aggregation. On the other hand, the same suspension of blood cells, when exposed to the influence of a powerful electric field (approximately $100 \mathrm{~V} . / \mathrm{cm}$.$) , rapidly forms macroscopically visible$ aggregations of red blood cells, quickly settling to the bottom. In this way, all erythrocytes are separated from the supernatant sugar solution in a few minutes.

The following interpretation of this surprising phenomenon is suggested. The erythrocytes contain a solution of different electrolytes (for example, $\mathrm{KCl}, \mathrm{K}-\mathrm{Hb}$, etc.) and are, therefore, conductors. When suspended in a solution of a non-electrolyte, they form a dispersion of small conductors in a nonconducting medium. In a strong electric field these small conductors become oriented by electric induction. The erythrocytes become dipoles, the opposite poles of which attract each other, and form chain-like aggregates. In accordance with Stokes's law, sedimentation-rates of such red-cell aggregates will be greater than those of single non-polarized blood cells.

Experimental. Human citrated blood is centrifuged, the plasma decanted, and the erythrocytes twice washed with an isotonic sucrose solution. Then the erythrocytes are suspended in isotonic sucrose solution. The suspension is poured into a U-tube (length about $10 \mathrm{~cm}$., diameter about $0.5 \mathrm{~cm}$.). The suspension in both limbs is covered with an isotonic solution of ghucose, which has a much smaller specific gravity than the isotonic sucrose solution. Metal electrodes are dipped in the glucose solution in both limbs of the U-tube, and 500-1,000 V. imposed on the electrodes. After approximately a minute, the red cells have formed clots, which rapidly sink to the bottom of the tube. When the current is stopped, the sedimented erythrocytes can be easily dispersed again by shaking. The electric aggregation of blood cells is, therefore, reversible, and in this respect analogous to the reversible coagulation of some colloids.

Department of Industrial Medicine

K. TEIGE

Z. STARÝ and Hygiene, Praha. 